\title{
The Understanding and Explanation of Sport: The Importance of Methodology
}

\author{
Almada F, Fernando C, Lopes $\mathrm{H}$ and Vicente $\mathrm{A}^{*}$ \\ Sports Sciences Department, Portugal
}

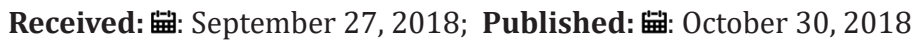

*Corresponding author: António Vicente, Sports Sciences Department, Convento de Santo António, 6201-001 Covilhã, Portugal

\section{Introduction}

Sport, like any other phenomenon, can be seen from many points of view, aiming at multiple objectives and, of course, with very different consequences (effects) and efficiencies. When we want to use sport as a factor of health, education, leisure or entertainment, or one of the many other functions for which sport can contribute in a powerful and competent way, we must overcome the still dominant tendency to consider it as a set of gestures or techniques that if often repeated "will have beneficial effects" (if it has effects, even if placebo, some will be positive but others will necessarily be negative, which we invariably forget). Understanding and being able to explain how the action tool SPORT works is fundamental so that we can define the active components, the dosages to be used, the contraindications that may have, etc. The mere accumulation of data and other products of more or less complicated investigations does not guarantee, at the outset, greater efficiency in the use of sport for defined and explicit purposes and objectives (and not mere generic intentions based on habits and customs, or empiricism) with the excuse "sport is good" [1].

Regarding the previous statement, we must be aware that change (the so famous "change" of which all speak vaguely and with generic bases - in such a way that it has almost become grotesque to speak of it) has already occurred and consolidated in broad terms. The "old world" (which existed even twenty or thirty years ago) is today "out of date". Certainly, in addition to the changes that are visible (which go unnoticed because we consider them to be "normal") at the technological level and even in lifestyles, they implied, and continue to imply, profound adaptations at a strategic, sociological, operational, relational, institutional levels, among others. In the impossibility of addressing all these issues in the small space of an article (but leaving the reader aware of the importance of a broader and more integrated vision), we will present some typical examples of changes in the understanding and explanation of sport, and its implications on the management of its activities (in the field of health, education, leisure, etc., in an aggregated form of all these aspects) [2].

The ability to measure, to treat data, to accumulate huge databases and to relate them, imposes global and integral changes. But data and information are nothing more than that: data and information. Today we possess them in abundance, and even in excess, over Man, Space, Earth, the physiological, mechanical, and psychological parameters of movement, atoms, matter, about almost everything. But they must be treated and integrated ("digested") so that they can be transformed into understanding, explanation of phenomena, knowledge, sapience and even wisdom [3]. Only in this way do they become useful and can be used (unlike databases and information that if not used are just a cost and even pollution that distracts and hides what is important). We'll show how some of these data and information that today also abound in sport, exercise and health, sports entertainment and its complements in multiple forms of social communication (comment, foresight, opinion, etc.), in health, in leisure and in many other ways, can be transformed into something useful and interesting allowing gains in understanding, explanation and evaluation of sports phenomena. We go beyond the mere description or expression of unsupported, or even foolish, comments and opinions. In this way, we complement some of the positions produced in our research, which are different perspectives and strands of the same view about Sport, in documents such as Almada et al. (in press), Almada et al., Almada et al. or in the "Tratado sobre Desporto" (English version: "Treaty on Sport").

\section{Some Applied Examples}

"Controlling a situation" is always a relative position (in relation to the pursued objectives, the available means, the oppositions encountered, etc.) that can be "solved" through organized and systematized work (structured and planned), or by mere accumulation of experiences that happen at random or in the flavor 
of sensitivities and impulses. Naturally the strategies used influence the obtained yields and the risks, as well as the range and depth of the knowledge that can be reached. Thus, for example, if we "live" from "sensitivities" and casual personal experiences, it is not easy to accumulate and transmit knowledge and perceptions, and to live on empiricisms that may be detailed and profound but can hardly be measured and transmitted in a broad way and without the commitment of some more sensitive people [4]. On the other hand, a treatment of knowledge in an organized and systematized way allows (imposes) already an accumulation of knowledge that can be more easily studied and transmitted, as well as the possibility of refuting (science, therefore, according to Karl Popper) and of confronting different positions to make visible the advantages and problems of each of the options. Let us present some examples of how to operate this option: In Tennis, in Football and in Judo.

\section{In Tennis}

The Serve Illustrating the problem exposed at the beginning of this article we will analyze the serve in tennis. In this sense we will consider, as an example, a set of variables that are the premises of the logical reasoning that we are going to carry out (naturally there will be many other variables and each of them can be deployed at many levels) [5].

\section{Premises:}

Time the Ball Takes Until it is Out of Reach of the Receiving Player: Today the average serves speed of a good player according ATP is $203 \mathrm{~km} / \mathrm{h}$. Therefore, there is about 0.36 seconds (s) since the ball is hit by the server until it can be returned.

a) Comment: The serve speed has evolved over time. According to ATP Leaderboards the 11 most-rated players in serve can reach speeds between $203 \mathrm{~km} / \mathrm{h}$ and $217 \mathrm{~km} / \mathrm{h}$, which equals ball travel times between 0.33 and 0.40 s (see the formula distance $=$ speed $\mathrm{x}$ time).

Distance Travelled by the Ball Until it is Hit by the Player who is Receiving it: Example: the ball travels about $20 \mathrm{~m}$ (we have $\mathrm{d}=20 \mathrm{~m}, \mathrm{v}=210 \mathrm{Km} / \mathrm{h}, \mathrm{t}=0,34 \mathrm{~s}$ ).

a) Comment: According to ATP statistics is in the "middle zone" that the highest percentage of serves are received, for which we considered, in the example, the distance of $20 \mathrm{~m}$ from the serve zone until the response.

Distance that the Opposing Player must Travel to Return the Ball - To return the ball the player who receives the serve usually has to move.

a) Comment: The speed of the player's movement varies according to the direction of the movement and the preparation to move (relation center of mass / support base), among others.

Reaction Time (Time Between Stimulus and Beginning of Response) - In a complex reaction let's consider about 0.55s.

a) Comment: The simple reaction time referenced in the literature are around $0.2 \mathrm{~s}$, however when it comes to complex reaction times values go up quite a lot between 0.31 and $0.65 \mathrm{~s}$, according to some studies (Davranche et al. 2006; Brito et al. 2011; O'Donovan et al. 2006; Mori et al.). When specific stimuli of the sport are used (through video situations), experienced sportsmen will have complex times around 0.55s / 0.70s or more.

Amplitude of Movement to Gain Speed on the Stroke - We must consider the time in which the player speeds up the racket to accumulate kinetic energy for the stroke.

a) Comment: The time the player receiving the serve has available for the response conditions the ability to respond, in speed and in accuracy [6].

\section{Reasoning:}

\section{If we articulate premise 1) with 2):}

In the Table 1 we can see the relation between the serve speed and the time that the ball takes to cover the distance of $20 \mathrm{~m}$, until it is within the reach of the receiver (Table 1).

Table 1: Relation between the speed at which the ball is hit and the time it takes to go through $20 \mathrm{~m}$.

\begin{tabular}{|c|c|}
\hline Speed $(\mathbf{k m} / \mathbf{h})$ & Time to go through $\mathbf{( d )}=\mathbf{2 0 m} \mathbf{( s )}$ \\
\hline 170 & 0,42 \\
\hline 180 & 0,40 \\
\hline 200 & 0,38 \\
\hline 210 & 0,36 \\
\hline 220 & 0,34 \\
\hline 230 & 0,33 \\
\hline 240 & 0,31 \\
\hline
\end{tabular}

If we articulate premise 1) (Given the Treatment in the Previous Reasoning) with 4):

In this isolation the tennis game is not possible because players would never catch the ball in a serve because the means of the reaction times are higher that the time that the ball takes to travel the distance to the other side of the field. But there are other variables to consider. The tennis player is required to anticipate his response by reading the server movement. And in the analysis of the response to the serve, in addition to the reaction time, we must also consider the time of displacement until the interception of the ball, the time of accomplishment of the beat, among other times. So, as we mentioned earlier, in addition to the strategy of trying to hit the serve with the greatest speed possible, it is necessary to play in the balance between the time the ball takes to travel the distance to the other field and also the time that the receiver will take to realize where will the serve go and the time it will take to move until respond the serve. In the background is the time differential between the server $(t)$ and the receiver $\left(t^{\prime}\right)$, where it is important to find the most favorable balance between these times [7].

Tennis is not just a relationship of players with a ball and rackets. It is an integrated dialogue between them. To understand it, it is not enough to know a set of techniques. There are innumerable premises that we could consider and various possible 
combinations. For this we have models of interpretation of the phenomenon (Almada et al.). According to the Taxonomy proposed by Fernando Almada, we can define a coherence for the activity and thus select the variables that may be more relevant not only for the understanding of tennis but also to intervene at the level of micro (training) and macro management (organizational structure). According to the Taxonomy proposed by Fernando Almada (Almada et al.), the Direct Confrontation Sports privileges the dialogue with the opponent through a direct confrontation that is usually carried out through an interposed object. In a simplified way, the model can be represented by $\mathrm{t} \leq \mathrm{t}$ ' ( $\mathrm{t}$ represents the attacker's action time, and $t^{\prime}$ the defender's time). The effectiveness of the action of the sportsman is about intervening in the times differential between these two times and not in playing with each of them independently. The ability to know the variables at play is fundamental to make the whole process profitable (at micro and macro levels), trying to understand the interactions that the variables establish with each other (as we presented above), in order to manage them in the best possible way, and taking into account not only the characteristics of the players involved but also the context [8].

Direct Confrontation (i.e. direct confrontation sport): results from the strategy followed by each of the players and their attention is focused on the changes that occur in the opponent which will condition (request) the changes and adaptations that the player will do in his action.

In Football (Soccer / Association Football) Passing: Let us now consider a passing situation in football (also known as soccer or association football). Also, in this example we'll consider a set of variables that are the premises of the logical reasoning that we are going to carry out (as already mentioned there are many other variables in play and each of them can be unfolded, but we'll try to be objective and synthetic).

\section{Premises}

a) Time the ball takes from the passing player until the teammate who will receive it $-60 \mathrm{~km} / \mathrm{h}$ is an acceptable reference speed for passing (Vicente et al.), and $20 \mathrm{~m}$ is also an acceptable distance to be covered by the ball in this situation. This means that the ball takes about $1.2 \mathrm{~s}$ to travel the $20 \mathrm{~m}$ between the two players.

b) Comment: The ball speed in a pass depends on aspects such as the distance to go through, the position of eventual opponents, the precision of who passes, the reception ability of the player who will receive it, the type of floor (if the grass is wet or not), among others. At $40 \mathrm{~km} / \mathrm{h}$ the ball travels $11.1 \mathrm{~m}$ in each second (Almada et al.).

c) Distance travelled by the ball until being received by the teammate - Acceptable distances that the ball can travel vary between $1 \mathrm{~m}$ and $40 \mathrm{~m}$.

d) Comment: In its course the ball can pass through elements of both teams that can interfere in its trajectory. There are still to consider the previous and following situations of the game. e) Distance that the opposing player must go through to intercept the ball - The game is in motion, we must consider the movements and intentions of the players.

f) Comment: The players speeds vary with the direction of the displacement, the previous preparation to move (relation center of mass / base of support), having or not the ball, among other. For example, running backwards or to the side is usually slower than forwards. FIFA data from the last World Cup presented Cristiano Ronaldo as the fastest player with a top speed of $34 \mathrm{~km} / \mathrm{h}$.

g) Reaction time (time between stimulus and beginning of response) - In a complex reaction let's consider about $0.55 \mathrm{~s}$.

h) Comment: As we presented in the Tennis example, also in Football we must consider complex reaction times (varying between 0.31 and $0.65 \mathrm{~s}$ ), and in ecological football situations for experienced sportsmen between $0.55 \mathrm{~s}$ and $0.70 \mathrm{~s}$ or more.

i) Windows of opportunity - coordination between whoever passes and who receives the ball is essential for the success of the action.

j) Comment: Often a pass is not successful because not only the coordination between who passes the ball and who would receive it was not the best but also because the action of players from both teams were poorly integrated. After all, it should be decided not by the pass that most easily reaches the partner, but by the one that also reaches the partner and enables a more favorable game sequence (Almada et al.).

\section{Reasoning}

Also, here in this example, we will follow the logic presented for Tennis.

a) If we articulate premise 1) with 2):

b) In the Table 2 we can see the relation between the ball speed and the time that it takes to cover the distance of $20 \mathrm{~m}$.

Table 2: Relation between the ball speed and the time it takes to go through $20 \mathrm{~m}$.

\begin{tabular}{|c|c|}
\hline Speed $\mathbf{( k m} / \mathbf{h})$ & Time to go through $(\mathbf{d}) \mathbf{2 0} \mathbf{m}(\mathbf{s})$ \\
\hline 10 & 7,19 \\
\hline 20 & 3,60 \\
\hline 30 & 2,40 \\
\hline 40 & 1,80 \\
\hline 50 & 1,44 \\
\hline 60 & 1,20 \\
\hline 70 & 1,03 \\
\hline
\end{tabular}

c) If we articulate premise 1) with 3) and 4):

d) In the net Table 3 we can see the relation between the defender displacement speed and the time that he takes to cover the distance of $5 \mathrm{~m}$. 
Table 3: Relation between the defender speed and the time he takes to go through $5 \mathrm{~m}$.

\begin{tabular}{|c|c|}
\hline Speed $(\mathbf{k m} / \mathbf{h})$ & Time to go through $(\mathbf{d}) \mathbf{5 m} \mathbf{( s )}$ \\
\hline 5 & 3,60 \\
\hline 10 & 1,80 \\
\hline 15 & 1,20 \\
\hline 20 & 0,90 \\
\hline 25 & 0,72 \\
\hline 30 & 0,60 \\
\hline 35 & 0,51 \\
\hline
\end{tabular}

Also in this football situation so that the defender can intercept the ball, for shorter distances, he will be forced to anticipate his action by reading the movement of the passer, since if we add the averages of reaction time and time to go through the reference space, these times are longer than the time the ball takes to travel the space to the possible point of interception / cut.

Passing is getting the ball to a teammate before an opponent can intercept it. Thus, if the time $(\mathrm{t})$ that the ball takes to travel the space until the player that receives it is greater than or equal to the time that the defender $\left(\mathrm{t}^{\prime}\right)$ takes to travel the space until the possible point of interception, the ball is intercepted and the pass is missed. On the contrary, if $t$ is less than $t^{\prime}$ the ball passes and the pass is successful. Here too the Taxonomy proposed by Fernando Almada (Almada et al.) helps us to understand and intervene. According this Taxonomy Collective Sports privilege the division of labor by different elements of a group, implying therefore the performance of specific functions and the domain of the dynamics of its coordination's (group dynamics). The model can be represented in a simplified form by $t \geq t^{\prime}$ ( $t$ being the time of the offensive action (including the ball) and $t$ ' the time of the defensive action), so $t$ and $t^{\prime}$ represent something different than $t$ and $t^{\prime}$ no tennis (in a more complex way we can assume in Collective Sports a model of $\sum t \geq \sum t^{\prime}$ in order to consider the various elements of the team) [9].
In a Collective Sport there are more teammates who can be possibilities to receive the pass, as well as more opponents who can seek to intercept the ball, combinations of variables (player skills) must be seen as a whole.

It is essential to make an analysis of each of the times $(t$ and $t^{\prime}$ ) by unfolding them at various times (time of movement, time of the ball in contact with the foot, time of the ball travel, time of reading the opponent's movement, decision making, displacement time, among others). It is essential not to lose the notion of the functioning of the whole trying to understand the interactions that the variables establish between each other to manage them in the best possible way considering the characteristics of the intervening sportsmen but also the context. Training is not only about learning to master football techniques.

The action of the collective, of the team (for that reason Collective Sport) results from the strategy followed by each one of the players and their attention is focused on the changes that occur in the opponents (at least in the most directly involved in the action), trying to deregulate their times, but also in the team itself trying to optimize the times obtained in their relationship (with or without ball) which will condition (request) the changes and adaptations that the player will make in his action. In this sense, playing football is not a relationship with the ball but the dialogue / opposition of two groups. Note: although the model in this Taxonomy is the same in its more simplified form for Direct Confrontation Sports and for Collective Sports, it is evident that in the unfolding of the variables we will have very different factors which leads to very different requests and adaptations (at physiological, psychological, technological, and other levels) [10].

\section{In Judo (Combat Sport)}

In Judo, the reasoning is identical, but again using the Taxonomy proposed by Fernando Almada the (very simplified) model is $\mathrm{F}$ $\leq F^{\prime}$, not only because of the difficulty in this modality to measure times but also because it is a more instinctive link of the combat phenomenon (shiai) and different forms of training (randori, uchikomi, etc.).
1.

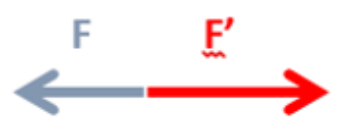

\section{$\mathrm{F}^{\prime}$ is greater than $\mathrm{F}$ - wins. But sometimes $\mathrm{F}^{\prime}$ unbalances and gets smaller, in these moments $\mathrm{F}$ can win.}

2.

$\mathrm{F}$ is always smaller than $\mathrm{F}^{\prime}$. But if well applied (direction perpendicular to the direction of $\mathrm{F}^{\prime}$ ) the force $F^{\prime}$ imposes its will to F? How?

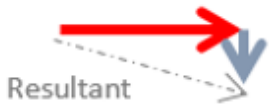

\section{Although much smaller F leads F' maintain its direction perpendicular to $\mathrm{F}^{\prime}$.}

Figure 1: How to obtain the predominance of $F$ over $F^{\prime}$ ? Two strategies and two logics in the requested technologies. 
In the oscillation between the predominance of $\mathrm{F}$ or $\mathrm{F}^{\prime}$, it becomes easy to define the strategies and technologies of operationalization, or even to define the "logic" and basic reasoning that support the activity as we can see schematically below where the analysis of the functional shows in a much more direct way than the ordinary description could do: (Figure 1)

a) In 1 and 2 different behaviors, strategies and ways of acting are requested (and therefore developed). 1 is about playing with the intensity of the forces. The strongest wins, even if it is stronger only for instants. One must take these moments to F or avoid them for F'.

b) In 2 we play with the ability to, even in risk situations, control the available means (even a lower force) and from there to control and lead the opponent. Different behaviors, strategies and ways of acting are requested (and thus developed). The training of the nervous control overlaps with the training of the intensity of the muscular capacities. They are different ways of fighting and consequently of being in life.

\section{Conclusion}

The break occurred. We live in conditions and in a context where efficiency is achieved with different strategies from what we were used to. The tendency to accumulate data, because they were rare and if we had them we would have the possibility to use them, "has gone." Today we have, or can get, all the data that we deem useful. Obviously always at some cost and efficiency must be considered (because saving data also has a cost (falling leaf plants perceive it - clever hem? - and prefer to lose them and then develop new ones). We can (should) focus our attention on strategies to develop and manage knowledge in an efficient way and request appropriate strategies. We can do it in a pleasing way by requesting (developing) abilities in sport, as a factor of health, or education, or leisure, or entertainment, among others., if we understand it to adapt it in an interesting way. We've showed, in a very succinct way, how to do it with different active components, different objectives, different dosages, etc., adapted to different sportsmen.

\section{References}

1. Almada F, Fernando C, Lopes H, Vicente A (2018) Understanding, Explaining and Managing Sport. In: Bento J (Eds.). Caring for the Common House: Nature, Life, Humanity. Opportunities and Responsibilities of Sports and Physical Education. Fortaleza, Brazil: State University of Ceará.

2. Almada F, Fernando C, Lopes H, Vicente A (2018) Operationalization of the Human Body Domain: A Structural and Functional Conception. J Phy Fit Treatment \& Sports 4(2).

3. Almada F, Fernando C, Lopes H, Vicente A (2018) Winning Efficiency. J Phy Fit Treatment \& Sports 4(2).

4. Almada F, Fernando C, Lopes H, Vicente A (2009) The Pass on Football. Serie 0 Futebol № 1. Mação: V.M.L.

5. Almada F, Fernando C, Lopes H, Vicente A, Vitória M (2008) The Breakthrough - Operational Strategy: The Systematics of Sports Activities. Torres Novas: VML.

6. Brito A, Silva C, Cid L, Ferreira D, Marques A (2011) Attention and reaction time in Shotokan karate practitioners. Journal of Asian Marciais Arts 6(1): 142-156.

7. Davranche K, Audiffren M, Denjean A (2006) A distributional analysis of the effect of physical exercise on a choice reaction time task. Journal of Sports Sciences 24(3): 323-329.

8. Mori S, Ohtani Y, Imanaka K (2002) Reaction times and anticipatory skills of karate athletes. Human Movement Science 21(2): 213-230.

9. O Donovan O, Cheung J, Catley M, MCgregor A, Strutton P (2006) An investigation of leg and trunk strength and reaction time of hardstyle martial arts practitioners. Journal of Sport Science and Medicine 5(CSSI): 5-12.

10. Vicente A, Lopes H, Fernando C (2016) Passing and Shooting in Football: Playing with the Ball... and the Others. $21^{\text {st }}$ Annual Congress of the European College of Sport Science (ECSS)-Crossing Borders Through Sport Science p. 514.

\section{ISSN: 2574-1241}

DOI: 10.26717/BJSTR.2018.10.001981

António Vicente. Biomed J Sci \& Tech Res

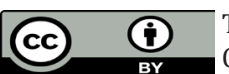

This work is licensed under Creative

Commons Attribution 4.0 License

Submission Link: https://biomedres.us/submit-manuscript.php

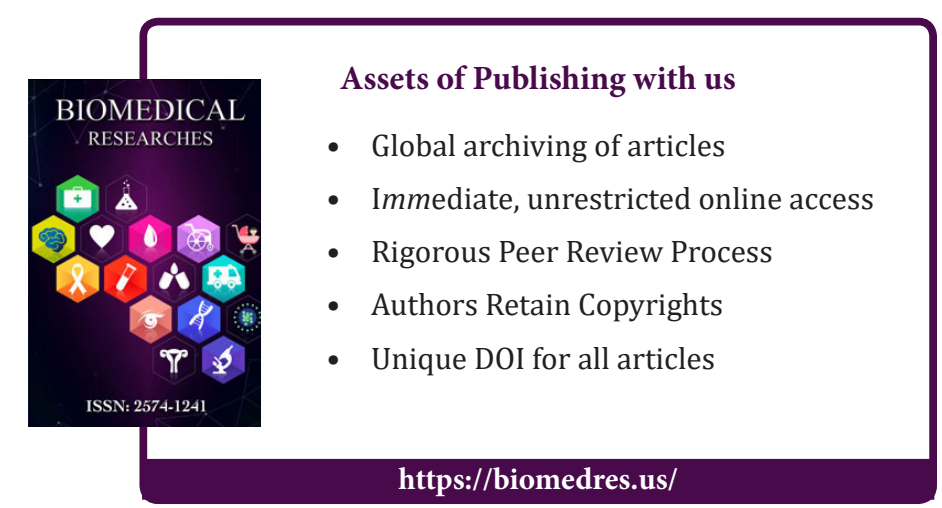

